\title{
NOVA TÉCNICA DE ALIMENTAÇÃO ENTERAL EM FÍSTULAS ESÔFAGO-JEJUNAIS
}

\section{NEW APPROACH TO ENTERAL NUTRITION IN ESOPHAGOJEJUNAL FISTULAE}

\author{
Guido Marks ${ }^{1}$ \\ Djalma José Fagundes, TCBC-SP ${ }^{2}$ \\ Francisco A Cevalos ${ }^{3}$
}

\section{INTRODUÇÃO}

Pacientes submetidos à gastrectomia total (GT) e à reconstrução esôfago-jejunal (REJ) podem apresentar deiscência da sutura esôfago-jejunal com conseqüiente fístula cutânea em até $8,2 \%$ nas suturas manuais ${ }^{1} \mathrm{e}$ de 2,8\% nas suturas mecânicas ${ }^{2}$. Costuma ocorrer no período compreendido entre o $4^{\circ}$ e $12^{\circ}$ dia de pós-operatório. A confirmação pode ser feita pela ingestão de azul de metileno e, mais recentemente, com a utilização da solução de sulfato de bário diluída a $50 \% \%^{3,4}$.

$\mathrm{O}$ tratamento conservador, com taxa de mortalidade em torno $19 \%$, inclui internação hospitalar prolongada com curativos freqüentes, antibioticoterapia e suporte nutricional. Uma nova intervenção operatória para sutura da deiscência e/ou exclusão do trânsito intestinal, em casos selecionados, está associada a taxa de mortalidade em torno de $64 \%$.

O suporte nutricional enteral por sonda é um recurso viável, entretanto, o refluxo jejuno-esofágico ocorre em 6,3\% e, com isto, contribui para a manutenção do processo 5 .

A nutrição parenteral prolongada é uma opção, mas apresenta também alta incidência de complicações e mortalidade, além dos custos hospitalares e dificuldades de manutenção dos cateteres em veias profundas 5 .

A última opção terapêutica que resta, esgotadas as possibilidades anteriores é uma nova intervenção, porém com elevado risco operatório e resultados precários.
O objetivo desta comunicação é apresentar uma alternativa de adaptação de cateter naso-enteral que proporcionaria uma proteção ao refluxo alimentar na zona da deiscência e que é considerado como um dos fatores para a persistência da fístula.

\section{TÉCNICA}

A proposta dos autores implica na confecção de um artefato que usa materiais de uso corrente em qualquer hospital: um cateter enteral para alimentação (adulto 12FR 120cm); um dreno laminar (Penrose) $\mathrm{n}^{\circ} 03,50 \mathrm{~cm}$; um fio de sutura inabsorvível número 0 de coloração preta; uma seringa de $20 \mathrm{ml} ; 20 \mathrm{ml}$ de solução salina de cloreto de sódio $0,9 \%$.

A montagem do equipamento começa pela colocação do cateter enteral através da luz do dreno laminar até atingir sua extremidade; este deve encamisar o cateter deixando cerca de $40 \mathrm{~cm}$ de dreno além do seu término e posteriormente amarrado, com fio inabsorvível, ao $1^{\circ}$ orifício de passagem do cateter enteral; a ponta do fio deve ser cortada a $2 \mathrm{~cm}$ do ultimo nó, para permitir a apreensão da pinça que conduzirá o conjunto nas manobras endoscópicas; duas amarrações ("reparos") adicionais eqüidistantes de $10 \mathrm{~cm}$ são realizadas distalmente à primeira, para segurança na fixação do dreno ao cateter enteral e servir de guia na introdução endoscópica do conjunto (Figura 1).

As manobras, sob sedação, para posicionar o conjunto confeccionado no trato digestório

1. Depto. de Clínica Cirúrgica, Universidade Federal de Mato Grosso do Sul

2. Prof. Adjunto da Disciplina de Técnica Operatória e Cirurgia Experimental do Depto. de Cirurgia da UNIFESP-EPM.

3. Médico do Serviço de Endoscopia SCOPE, Campo Grande, M.S.

Recebido em 15/04/2003

Aceito para publicação em 02/09/2003

Trabalho realizado pelo Departamento de Clínica Cirúrgica, Universidade Federal de Mato Grosso do Sul e Depto. de Cirurgia da UNIFESP-EPM. 


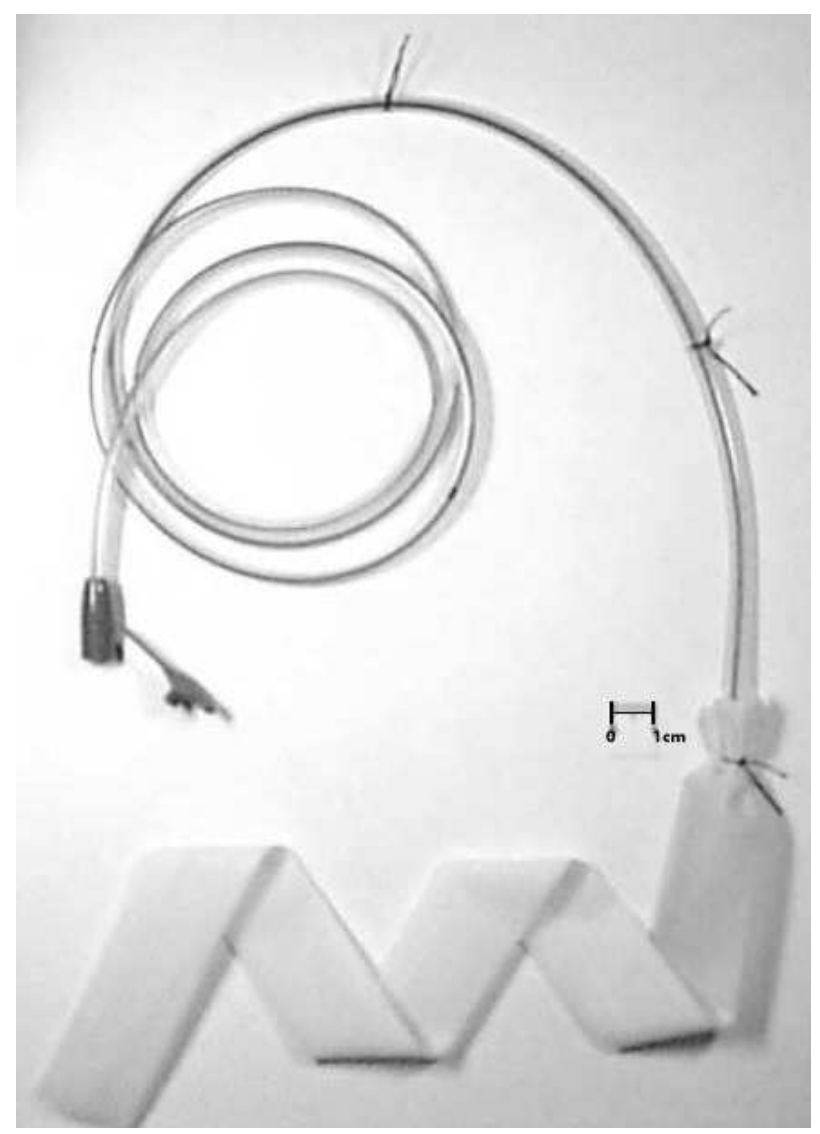

Figura 1 - Conjunto montado.

do paciente segue a seguinte sequiência: introdução do cateter pela narina ao mesmo tempo em que se efetua a introdução do endoscópio por via oral; sob controle visual observa-se o aparecimento do cateter na cavidade da orofaringe; apreende-se o conjunto com a pinça flexível de biópsia endoscópica, utilizando o primeiro fio longo de fixação do dreno laminar; condução do conjunto através do esfíncter superior do esôfago até atingir a região da anastomose esôfagojejunal; identifica-se o orifício interno do trajeto fistuloso, evitando-se manipulação excessiva ou traumática da região; identifica-se o lúmen jejunal e conduz-se o aparato por $10 \mathrm{~cm}$ além da anastomose; traciona-se cranialmente o endoscópio até identificar o próximo reparo com apreensão deste e condução caudal do conjunto em manobras sucessivas até o posicionamento do último reparoao nível da anastomose esôfago-jejunal (Figura 2); instila-se $20 \mathrm{ml}$ da solução salina de cloreto de sódio $0,9 \%$ pelo cateter e distende-se o dreno laminar até o posicionamento (50 cm de comprimento) avançado no jejuno; a observação endoscópica direta do início da migração do dreno laminar (instilação de $20 \mathrm{ml}$ de solução salina) permite comprovar a sua permeabilidade e conferir o posicionamento adequado do conjunto. A alimentação enteral pode ser iniciada precocemente e mantida até a resolução da fístula.

\section{DISCUSSÃO}

O tratamento de escolha para o câncer de estômago proximal é a gastrectomia total nos estágios I e II, associado à linfadenectomia e sem esplenectomia ${ }^{6}$. A reconstrução de escolha é o procedimento em Y de Roux com alça jejunal, que apresenta melhor resultado a longo prazo.

A fístula esôfago-jejunal é uma complicação grave que acompanha este procedimento operatório. O tratamento conservador exibe índice de morbidade de até $17,8 \%$ e índice de mortalidade de $2,5 \%{ }^{9}$. A reintervenção operatória e anastomose intratorácica ${ }^{6}$ faz com que este último índice atinja níveis de 10,6 a $44 \%$.

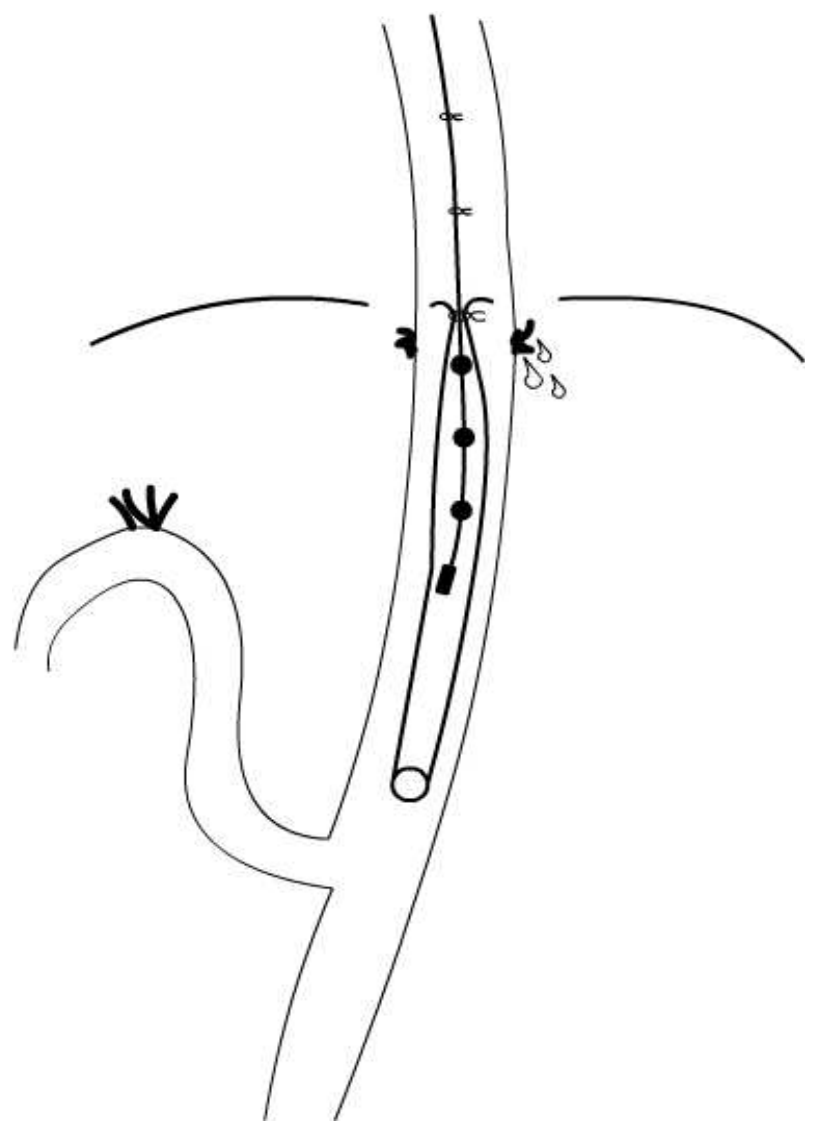

Figura 2 - Esquema do conjunto posicionado. 
Uma opção é a abordagem endoscópica do trajeto fistuloso e a colocação de um clipe metálico no orifício interno da fístula. Os resultados mostram baixa morbimortalidade, porém é de difícil realização técnica, exigindo equipamentos especializados ${ }^{5,6}$.

O tratamento conservador pela alimentação enteral através de uma sonda que ultrapasse a zona da deiscência é um procedimento bastante utilizado e de fácil execução. Contudo, o refluxo da dieta administrada através da sonda é tido como um fator de manutenção do débito da fístula. Considera-se que o refluxo do líquido entérico, que resulta da estase da alça exclusa na confecção do Y de Roux, seja também outro fator de manutenção da fístula ${ }^{5,6}$.

A proposta da associação do cateter enteral para alimentação envolvida por um dreno laminar é baseada na premissa teórica que este protegerá a área da deiscência de um fluxo de alimentos e de líquido entérico retrógrado. O fato deverá favorecer o processo cicatrização pela diminuição ou ausência do fator irritativo destes líquidos.

O procedimento é de fácil execução podendo ser realizado em qualquer hospital que disponha de um serviço de endoscopia digestiva. Os custos dos materiais utilizados são baixos e são de fácil aquisição.

Para uma aplicação em larga escala o procedimento merece ser estudado do ponto de vista experimental em animais. A aplicação deste procedimento em seres humanos, com as devidas cautelas de ordem ética, é factível uma vez que trata-se de uma variação de procedimentos já largamente utilizados e com intercorrências mínimas. A validade do modelo teórico proposto depende de uma avaliação criteriosa a médio e longo prazo. Resultados preliminares da aplicação do procedimento mostraram-se bastante promissores.

\begin{abstract}
Background: A new procedure for the treatment of esophageal fistula, mainly associated to the ebb esophagojejunal in patients submitted to the total gastrectomy and reconstruction with loop jejunal Rouxen-Y anastomosis is present. Methods: The method is based in the use of "probe standard enteral prolongated with drain to laminate adapted in extremity", which results in advanced positioning inside the jejunum, making the administration of enteral nutrition possible and impeding ebb esophagojejunal. Results: The authors discuss the theoretical advantages of the procedure and they suggest that the treatment of esofagic fistula with probe prolonged enteral would be suitable in the treatment of the fistula esophagojejunal by preventing the ebb esophagojejunal, which would result in smaller period of duration of the fistula esophagojejunal and it would prevent the high mortality rate. Conclusions: Preliminary studies demonstrated that this is a technically easy, low cost procedure through the endoscopic use. A prospective evaluation for morbility and mortality related to the method is needed.
\end{abstract}

Key Words: Fistula; Digestive system fistula; Gastroesophageal reflux; Anastomosis, Roux-en-Y; Enteral Nutrition; Postoperative complications, Endoscopy.

\section{REFERÊNCIAS}

1. Isozaki H, Okajima K, Ichinona T, et al. - Risk factors of esophagojejunal anastomotic leakage after total gastrectomy for gastric cancer. Hepatogastroenterology, 1997, 44(17): 1509-1512.

2. Pol B, LeTreut YP, Hardwigsen J, et al. - Mechanically stapled esophagojejunostomy. Results of a prospective series of 176 cases. Hepatogastroenterology, 1997, 44(14): 458-466.
3. Schardey HM, Joosten U, Finke U, et al. - The prevention of anastomotic leakage after total gastrectomy with local decontamination. A prospective, randomized, double-blind, placebocontrolled multicenter trial. Ann Surg, 1997, 225(2): 172-180.

4. Gollub MJ, Bains MS - Barium sulfate: a new (old) contrast agent for diagnosis of postoperative esophageal leaks. Radiology, 1997, 202(2): 360362. 
5. Lang H, Piso P, Stukenborg C, et al. - Management and results of proximal anastomotic leaks in a series of 1114 total gastrectomies for gastric carcinoma. Eur J Surg Oncol, 2000, 26(2): 168-171.

6. Roy-Choudhury SH, Nicholson AA, Wedgwood KR, et al. - Symptomatic malignant gastroesophageal anastomotic leak: management with covered metallic esophageal stents. AJR Am J Roentgenol, 2001, 176(1):161-165.
Endereço para correspondência: Guido Marks/Djalma José Fagundes Disciplina de TOCE - Depto Cirurgia UNIFESP-EPM

Rua Botucatu , 720 CEP 04023-900 - Vila Clementino - São Paulo- SP E-mail:djfagundes.dcir@epm.br 